\title{
Fractional variational principles and their applications
}

\author{
Dumitru Baleanu ${ }^{1,2 *}$ \\ ${ }^{1}$ Department of Mathematics and Computer Sciences, Faculty of Arts and Sciences, Cankaya University, \\ 06530, Ankara, Turkey. \\ ${ }^{2}$ Institute of Space Sciences, P.O.BOX, MG-23, R 76900, Magurele-Bucharest, Romania.
}

\begin{abstract}
Variational calculus and fractional calculus have played a significant role in various areas of applied sciences such as, among others, Physics, Engineering and Economics. This topic is deeply connected to the very recent developments in theoretical aspects and especially in the numerical schemes of fractional differential equations. Based on $1+1$ field formalism, a new fractional Lagrangian and Hamiltonian formalisms are presented within the Riemann-Liouville fractional derivatives and the an-harmonic oscillator is analyzed. This formalism can be applied to analyze the control problems as well as for the fractional quantization procedure.
\end{abstract}

Copyright line will be provided by the publisher

\section{Introduction}

Fractional derivatives [1,2] started to play an important role in many branches of science and engineering. Various applications of fractional calculus in physics [3-6], robotics [7] and control theory [8,9] have been obtained. For almost all systems that contain internal damping, the traditional energy-based approach cannot be used to obtain the correct equations describing the behavior of a nonconservative system. By using fractional calculus techniques, the Lagrangian and the Hamiltonian equations of motion for non-conservative systems can be obtained [5,6,8]. As it is known, a characteristic of the Hamiltonian formalism for non-local theories is that it contains the Euler-Lagrange equations as Hamiltonian constraints.In this paper the fractional Lagrangian to start with is $L_{f}\left(q(t),{ }_{a} \mathbf{D}_{t}^{\alpha} q(t)\right)$ and it becomes the classical Lagrangian for $\alpha=1[5,6,8]$. Here ${ }_{a} \mathbf{D}_{t}^{\alpha} q(t)$ denotes the left Riemann-Liouville fractional derivatives defined as follows [1,2]

$$
{ }_{a} \mathbf{D}_{t}^{\alpha} q(t)=\frac{1}{\Gamma(n-\alpha)}\left(\frac{d}{d t}\right)^{n} \int_{a}^{t}(-\tau+t)^{n-\alpha-1} q(\tau) d \tau,
$$

where $\Gamma(\alpha)$ denotes the gamma function [2]. The fractional derivatives contain memory and they have more restrictive properties compared to the classical ones [1,2]. Let us take an analytic function $q(t)$ and $p(t)=H(t-a)$, where $H(t)$ is the Heaviside function. Using the fractional Leibniz rule and the formula for the fractional differentiation of the Heaviside function we obtain [2]

$$
{ }_{a} \mathbf{D}_{t}^{\alpha} q(t)=\frac{(t-a)^{-\alpha}}{\Gamma(1-\alpha)} q(t)+\sum_{k=1}^{\infty}\left(\begin{array}{c}
\alpha \\
k
\end{array}\right) \frac{(t-a)^{k-\alpha}}{\Gamma(k-\alpha+1)} q^{(k)}(t)
$$

under the assumption $t>a$.

\section{A new fractional Hamilton formalism}

For a given classical Lagrangian $L(q(t), \dot{q}(t))$ the corresponding fractional one is obtained by replacing the classical derivatives with the fractional ones. The existence of the infinite higher order derivatives in (2) and in the fractional Lagrangian $L_{f}\left(q(t),{ }_{a} \mathbf{D}_{t}^{\alpha} q(t)\right)$ lead us to an important issue regarding the form of the corresponding Euler-Lagrange and Hamiltonian equations. The solution is to use the formula (2) and to consider the dynamical variable $q(t)$ as a $1+1$ dimensional field $Q(x, t)$ such that the following chirality condition $\frac{d Q(x, t)}{d t}=\partial_{x} Q(x, t)$ is valid [10]. $Q(x, t)=q(x+t)$ assures the one-toone correspondence between $q(t)$ and $Q(x, t)$ [10]. Ostrogradski's coordinates are defined as follows [10]

$$
Q^{(n)}(t)=\left.\left(\partial_{x}\right)^{n} Q(x, t)\right|_{x=x_{0}}
$$

where the discontinuity curve $x_{0}(t)=x_{0}$ is a constant. By making use of the inverse relation provided by the Taylor expansion around $x=x_{0}$ we obtain

\footnotetext{
* Corresponding author E-mail: dumitru@cankaya.edu.tr, Phone: +90312 284 4500, Fax: +90 3122898612
} 


$$
Q(x, t)=\sum_{n=0}^{\infty} \frac{\left(x-x_{0}\right)^{n}}{n !} Q^{(n)}(t) .
$$

The chirality condition is given by $\dot{Q}^{(n)}(t)=Q^{(n+1)}(t)$. The Ostrogradski's momenta $P_{(n)}(t)$ are define as in the following

$$
P_{(n)}(t)=\int_{-\infty}^{\infty} d x \frac{\left(x-x_{0}\right)^{n}}{n !} P(x, t) .
$$

From (5) the form of $P(x, t)$ is given by

$$
P(x, t)=\sum_{n=0}^{\infty} P_{(n)}(t)\left(-\partial_{x}\right)^{n} \delta_{R}\left(x-x_{0}\right) .
$$

By using (6) the expression for $P_{(n)}(t)$ becomes

$$
P_{(n)}(t)=\sum_{m=n}^{\infty}\left(-\partial_{t}\right)^{m-n} \frac{\partial L_{f}[Q](t)}{\partial Q^{(m+1)}(t)} .
$$

As a result, the fractional Euler-Lagrange equation becomes $\dot{P}_{(0)}(t)=\frac{\partial L_{f}[Q](t)}{\partial Q^{(0)}(t)}$ and the Hamilton's equations are given by

$$
\dot{P}_{(n)}(t)+P_{(n-1)}(t)=\frac{\partial L_{f}[Q](t)}{\partial Q^{(n)}(t)}, n \in N
$$

Here $L_{f}[Q](t)$ is obtained from $L\left(q(t),{ }_{a} \mathbf{D}_{t}^{\alpha} q(t)\right)$ by taking into account that $Q(x, t)=q(x+t)$. The action can be constructed by using the above Hamiltonian, therefore we can quantize the theory. In this manner a physical interpretation for the fractional action is obtained. The effect of fractional derivative is given by $\alpha$ which appears in the decomposition of the fractional derivative (2). We mention that this formalism differs from the classical ones $[5,6,8]$ based by replacing the classical derivatives by the fractional ones and by using the fractional integration by parts. This new formalism can be applied for the fractional control problems and it is an alternative method to the fractional variational principles developed in [5, 6, 8].

\subsection{Example}

For the an-harmonic oscillator of unit mass and frequency $\omega$, the classical Lagrangian

$$
L=\frac{\dot{q}(t)^{2}}{2}-\frac{\omega^{2} q^{2}(t)}{2}-\frac{\omega^{2} q^{3}(t)}{2}
$$

can be fractionalized as

$$
L_{f}=\frac{\left({ }_{a} \mathbf{D}_{t}^{\alpha} q(t)\right)^{2}}{2}-\frac{\omega^{2} q^{2}(t)}{2}-\frac{\omega^{2} q^{3}(t)}{2} .
$$

According to the new formalism presented before we obtain the fractional Euler-Lagrange equation as $\dot{P}_{(0)}(t)=\frac{(t-a)^{-\alpha}}{\Gamma(1-\alpha)}{ }_{a} \mathbf{D}_{t}^{\alpha} Q(t, x)$ and the fractional Hamilton's equations have the form $\dot{P}_{(n)}(t)+P_{(n-1)}(t)=\left({ }_{a} \mathbf{D}_{t}^{\alpha} Q(t, x)\right)\left(\begin{array}{c}\alpha \\ n\end{array}\right) \frac{(t-a)^{n-\alpha}}{\Gamma(n-\alpha+1)}, n \in N$

Acknowledgements The author would like to thank J.J. Trujillo and M. Kirane for reading the manuscript and for valuable discussions.

\section{References}

[1] I. Podlubny, Fractional Differential Equations (Academic Press, San Diego CA, 1999), p. 88.

[2] A. A. Kilbas, H. H. Srivastava, and J. J. Trujillo,Theory and Applications of Fractional Differential Equations (Elsevier, The Nederlands, 2006), p. 90.

[3] D. Baleanu and S. I. Muslih, physica scripta 72, 119 (2005).

[4] R. Hermann, j. phys. g:part.phys. 34, 607 (2007).

[5] E. M. Rabei, K. I. Nawafleh, R. S. Hijjawi, S. I. Muslih, and D. Baleanu, j. math. anal. appl. 327, 891 (2007).

[6] F. Riewe, phys. rev. e 53, 1890 (1996).

[7] M. F. Silva, J. A. Tenreiro Machado, and A. M. Lopes, robotica 23, 595 (2005).

[8] O. P. Agrawal, j. math. anal. appl. 273, 368 (2002).

[9] O. P. Agrawal and D. Baleanu, j. vib. contr. 13, 1269 (2007).

[10] J. Gomis, K. Kamimura, and J. Llosa, phys. rev. d 63, art. no. 045003 (2001). 